NBER WORKING PAPER SERIES

THE DOMINANCE OF RETAIL STORES

\author{
Alexandre Ziegler \\ Edward P. Lazear \\ Working Paper 9795 \\ http://www.nber.org/papers/w9795 \\ NATIONAL BUREAU OF ECONOMIC RESEARCH \\ 1050 Massachusetts Avenue \\ Cambridge, MA 02138 \\ June 2003
}

The authors would like to thank Stefan Arping, Michel Habib, Sam Peltzman, Paul Romer and Alfonso Sousa-Poza for numerous valuable suggestions which greatly improved the paper. The views expressed herein are those of the authors and not necessarily those of the National Bureau of Economic Research.

C2003 by Alexandre Ziegler and Edward P. Lazear. All rights reserved. Short sections of text not to exceed two paragraphs, may be quoted without explicit permission provided that full credit including (C) notice, is given to the source. 
The Dominance of Retail Stores

Alexandre Ziegler and Edward P. Lazear

NBER Working Paper No. 9795

June 2003

JEL No. D40, D44

\begin{abstract}
Most items are sold to consumers by retail stores. Stores have two features that distinguish them from auctions. First, the price is posted and a consumer who values the good at more than the posted price is sold the good. Second, the sale takes place as soon as the consumer decides to buy. In contrast, auctions have prices that are determined ex post and the potential consumer must wait until the auction is held to buy the good. Consequently, auctions result in "false trading", where buyers sometimes pass up other valuable opportunities while waiting for the auction to occur or instead make undesired duplicate purchases. Retail stores dominate auctions when the good is perishable and/or becomes obsolete quickly, when the market is thin, and when close substitutes for the good are plentiful. These predictions are consistent with a number of observed phenomena.
\end{abstract}

\author{
Alexandre Ziegler \\ Ecole des HEC \\ University of Lausanne \\ Switzerland \\ aziegler@hec.unil.ch
}

Edward P. Lazear

Graduate School of Business

and Hoover Institution

Stanford University

Stanford, CA 94305-5015

and NBER

lazear@gsb.stanford.edu 


\section{The Dominance of Retail Stores}

Most goods are sold to consumers through retail stores, where a seller commits to sell at a given price and where the good is sold as soon as the consumer decides to purchase. However, it is commonly alleged that an auction should be run whenever the seller wants to maximize the proceeds from selling a good that potential buyers value differently. ${ }^{1}$ The dominance of auctions over stores is at variance with the real world observation that stores account for the vast majority of sales to consumers.

Stores have two advantages over auctions, which account for their prevalence. Stores avoid imposing waiting cost on consumers. In order to command a price premium above the reservation price of the seller, any form of auction requires several bids to accumulate before it is cleared. As a result, there is usually a certain waiting period between the time an auction is announced (when the good would actually be available for sale) and the time the winner is determined (when the good becomes available to the buyer). ${ }^{2}$ The second advantage that stores possess is that they eliminate some uncertainty that is inherent in auctions. Consider a buyer seeking to purchase a bottle of milk for his next breakfast. If milk were sold to consumers by auction, the consumer would not know whether he made the winning bid or not until the auction cleared. If he were to see another opportunity to purchase milk, either by store or auction, he would face a dilemma. If he does not purchase the milk there, he runs a significant risk of not having milk the next morning. On the other hand, if he does purchase milk in the store or chooses to bid on the second auction, he risks ending up with two bottles of milk. Stores eliminate the "false trading" that is created by auctions where some buyers who would agree to pay more than the market clearing price do not get the item and others who would be unwilling (ex post) to pay as much as the clearing price are forced to buy the good because they are locked in by the auction scheme.

Note that nothing in the definition of store requires a physical structure. An online store fits into the category of stores if it offers to sell at a posted price and provides delivery within a specified (generally short) time after the sale has been concluded.

\footnotetext{
${ }^{1}$ Milgrom (1987) shows that in a full-information setting, a take-it-or-leave-it offer of the seller to the highest valuation buyer extracts all the surplus. On the other hand, in the more realistic setting in which buyers' valuations are private information, a sealed-bid auction with a minimum price maximizes the seller's payoff among all possible exchange games.

${ }^{2}$ Of course, it is conceivable that actions are announced in advance of the good's availability with a closing at exactly the moment that the good becomes available. Such a strategy works in a deterministic world, but not in one where supply has a stochastic component. Most of retailing deals with goods where at least some component of good availability is random.
} 
The way auctions are designed in practice constitutes strong evidence of the relevance of this false trading problem. Because bidders typically do not wish to submit bids too long before the auction closes to avoid being locked in by the auction scheme, sellers typically choose to run auctions that close at a pre-specified time and not after a certain number of bids has been received. False trading also affects bidding behavior. Indeed, in the context of online auctions, it has been documented that a considerable proportion of bids arrive towards the end of the auction, a phenomenon commonly known as sniping (Pinker et al. (2002)). ${ }^{3}$

Intuitively, one would expect waiting costs and the risk of false trading to make the store a more appealing selling institution than auctions under most circumstances. This paper presents a formal model confirming this intuition. We model bidders' waiting costs and the risk of false trading by assuming that bidders having to wait for the auction to close will engage in a search process to purchase the good elsewhere. When such a purchase opportunity is found, a bidder that was originally planning to participate in the auction drops out. With fewer bidders participating, the seller's expected revenue from the auction falls. As a result, the costs imposed on bidders by the auction get internalized by the seller and he is more likely to sell the good using a store rather than an auction.

Our results show that the optimal choice of the store as opposed to the auction structure is driven by a combination of three factors: (1) the extent to which the good being sold is perishable, becomes obsolete quickly and/or the seller has a strong time preference, (2) the thickness of the market, and (3) the size of bidders' waiting costs and the extent to which alternatives to purchase the good elsewhere are available. More specifically, we establish the following results:

1. If the good is perishable, becomes obsolete quickly and/or the seller has a strong time preference, then the store is preferred. Intuitively, this effect arises because perishability and discounting make it more costly for the seller to wait for additional bidders before closing the auction. As a result, the optimal auction has a small expected number of bidders, and the store dominates.

2. If the market is thick in the sense that the rate of bidder arrivals is high, the auction is preferable. This effect arises because when the rate of bidder arrivals is high, the gain in expected selling price per unit time the seller waits before closing the auction is increased, making it worthwhile to wait for a large number of bidders.

\footnotetext{
${ }^{3}$ The observation itself does not necessarily imply that waiting costs are central. It is also possible that another sort of strategic behavior explains sniping.
} 
3. If bidders' waiting costs are high and/or substitutes for the good are readily available, then the store is preferred. This is because those buyers who arrive before the auction's closing time are reluctant to wait, and either leave or search for an alternative. As a result, the auction is unable to achieve the level of bidder participation required to command a premium over the posted price. In the context of this point, the effects of both elasticities of demand and supply can be analyzed. When either side of the market has high degrees of elasticity, goods will be sold in retail stores.

An important implication of our analysis is that the optimal way to sell similar goods can differ, depending on the environment. For example, the thickness of a market can have a decisive impact on the choice between store and auction, with thin markets using stores and thick markets auctions to sell the same goods. Furthermore, seemingly identical goods can be sold either through a store or an auction depending on the degree of availability of substitutes.

The remainder of the paper is organized as follows. Section 1 describes the store. Section 2 considers the properties of the optimal auction. Section 3 compares the two selling institutions and derives conditions under which each of those forms dominates. Section 4 concludes. Proofs of the propositions are in the Appendix.

\section{The Store}

Consider a risk-neutral seller trying to sell a single unit of an indivisible good using a store. He announces a listing price $p$ for the good; the first customer that agrees to pay the listed price gets the good.

Potential customers arrive according to a Poisson process with intensity $\lambda$, implying that the customer interarrival time is exponentially distributed with parameter $\lambda$. Each potential buyer $i$ has a valuation $v_{i}$ for the good, which is random, independent of other buyers' valuations and has distribution function $F$ and density $f$ with support $[\underline{v}, \bar{v}]$. The customer buys the good if $v_{i}>p$. If his valuation is below $p$, the good remains available for sale to another customer.

Let $\alpha>0$ denote the discount rate for this problem, which can be interpreted either as the seller's time preference, as the extent to which the item being sold is perishable, or as the speed with which it becomes obsolete. ${ }^{4}$ Let $0 \leq R<\bar{v}$ denote the seller's reservation utility if the good

\footnotetext{
${ }^{4}$ In the first interpretation, all amounts received by the seller are simply discounted at a rate $\alpha$. In the second and third interpretation, bidders' valuations for the good would be decaying exponentially through time, with a time-dependent distribution $F(v, t)$ rescaled so as to lie on the support $\left[e^{-\alpha t} \underline{v}, e^{-\alpha t} \bar{v}\right]$ (the same would apply
} 
goes unsold. In the spirit of dynamic programming, $R$ can be viewed as summarizing the entire future of the problem (except for the effect of obsolescence, perishability and discounting, which is captured by $\left.e^{-\alpha t}\right)$. It could represent either the utility the seller derives from consuming the good himself, or the expected revenue from a subsequent sale.

The store owner's problem is to set a price schedule $\left\{p_{t}\right\}$ that maximizes the expected revenue from the sale, $\Pi_{S}$. Given $p_{t}$ and the distribution of buyers' valuations, for any customer walking into the store at time $t$, the good will be sold with probability $1-F\left(p_{t}\right)$. Thus, the store's expected revenue is $p_{t}\left(1-F\left(p_{t}\right)\right)$. With probability $F\left(p_{t}\right)$, the good will not be sold, and the store owner receives the reservation utility $R$. Formally, the store owner's problem is to

$$
\max _{\left\{p_{t}\right\}} \Pi_{S}=\int_{0}^{\infty} e^{-\alpha t} \lambda e^{-\lambda t}\left(p_{t}\left(1-F\left(p_{t}\right)\right)+F\left(p_{t}\right) R\right) d t
$$

where $\lambda e^{-\lambda t}$ is the density of interarrival times and $e^{-\alpha t}$ captures the effect of perishability and discounting. With $R$ given, one can do no better than maximizing $p_{t}\left(1-F\left(p_{t}\right)\right)+F\left(p_{t}\right) R$ time by time, yielding the first-order condition

$$
1-F\left(p_{t}\right)-p_{t} f\left(p_{t}\right)+f\left(p_{t}\right) R=0
$$

which immediately implies that $p_{t}$ is a constant independent of time, $p^{*}$, satisfying the usual hazard rate formula, ${ }^{5}$

$$
p^{*}=R+\frac{1-F\left(p^{*}\right)}{f\left(p^{*}\right)}
$$

Using this solution, the store's maximum expected revenue, $\Pi_{S}$, is given by

$$
\Pi_{S}=\int_{0}^{\infty} \lambda e^{-(\alpha+\lambda) t}\left(p^{*}\left(1-F\left(p^{*}\right)\right)+F\left(p^{*}\right) R\right) d t
$$

Integrating,

$$
\Pi_{S}=\frac{\lambda}{\alpha+\lambda}\left(p^{*}\left(1-F\left(p^{*}\right)\right)+F\left(p^{*}\right) R\right)
$$

Note that since $p^{*}=R+\frac{1-F\left(p^{*}\right)}{f\left(p^{*}\right)}>R$, on has $p^{*}\left(1-F\left(p^{*}\right)\right)+F\left(p^{*}\right) R>R$ and therefore $\Pi_{S}>\frac{\lambda}{\alpha+\lambda} R$. As a result, there exists some $\alpha^{*}>0$ such that the seller derives more than his reservation utility from selling the good whenever $\alpha<\alpha^{*}$. This result, which will be important below, is summarized in the following proposition:

to the reservation utility $R$ ). Both of these cases can be handled with minor modifications of the formulation used below and yield similar conclusions.

${ }^{5}$ Lazear (1986) describes the optimal time path of prices when the seller faces uncertainty about the market for the item being sold. His results differ because in his model, the number of consumers is finite and they have common valuations. 
Proposition 1: Let $p^{*}=R+\frac{1-F\left(p^{*}\right)}{f\left(p^{*}\right)}$. Then, for all $\alpha<\alpha^{*} \equiv \lambda \frac{\left(p^{*}-R\right)\left(1-F\left(p^{*}\right)\right)}{R}$, the seller's expected utility from the sale, $\Pi_{S}$, is strictly greater than his reservation utility $R$.

Proposition 1 has the immediate implication that if the discount rate $\alpha$ is very high (either because the seller has a very high time preference, the good is highly perishable or becomes obsolete quickly), then the seller will not even attempt to sell the good and prefer to consume it himself. It follows trivially that attempting a sale is always optimal if $R=0$.

\section{The Auction}

\subsection{The Model}

Suppose now that the seller decides to sell the good using an auction. He announces a reservation price $p$ and the auction's closing time $T{ }^{6}$ Individuals who arrive before $T$ are allowed to submit bids for the good. At time $T$, the highest bidder gets the good at a price equal to the second-highest bid or the reservation price $p$, whichever is greater. ${ }^{7}$

Not all arrivals result in bids because the necessity of waiting until $T$ imposes an additional cost on bidders. This is captured by allowing alternative purchase opportunities that are idiosyncratic to each bidder to arrive exponentially at rate $\mu$ for each individual bidder engaged in the search process. ${ }^{8}$ In practice, the value of $\mu$ will depend positively on two factors, each having its own economic interpretation: on how important immediacy of the purchase is to bidders (with high direct waiting costs leading them to search for alternatives more intensively or to decide to purchase goods that are inferior to the one being auctioned), and on the extent to

\footnotetext{
${ }^{6}$ Although an auction with a fixed closing time is used in the analysis, the results below hold equally true under the alternative assumption that the auction closes after a certain number of bids has been received. This follows from the fact, established in proposition 2 below, that the seller's expected proceeds are increasing and concave both in the actual and in the expected number of bidders participating in the auction.

${ }^{7}$ Given the equivalence theorem for independent private value auctions (Vickrey (1961), Myerson (1981), Riley and Samuelson (1981)), this assumption is innocuous for the optimality of the auction as opposed to the store in the setting considered here, but simplifies the exposition as bidders bid their true valuation.

${ }^{8}$ This assumption is made for expositional convenience. All that is required for the results below to hold is that the probability that a bidder drops out from the auction be an increasing function of the time he must wait before the auction closes. However, it is worth noting that assuming an exponential distribution is not unrealistic. It corresponds to the limiting case of a situation in which searching bidders visit a certain number of places per unit time until the auction closes and there is a certain probability that they find a suitable good in each of these places. (Note that the good need not be strictly identical to the one being auctioned, as some bidders will choose to purchase an inferior alternative because they do not want to wait.)
} 
which substitutes for the good being auctioned exist (which, with some qualifications discussed below, would generally be high for commodity products and low for original artwork). Note that if many substitutes exist, then the probability that bidders find an alternative by time $T$ is high and so would be the risk of false trading if bidders chose to submit their bids as soon as they arrive at the auction (i.e., before searching for alternatives). ${ }^{9}$

The number of bidders that participate in the auction at time $T, N$, equals the number of bidder arrivals minus the number of bidders that have found an alternative by time $T$. Thus, as shown in Appendix A, $N$ follows a Poisson distribution with parameter

$$
\tilde{\lambda}(T)=\frac{\lambda}{\mu}(1-\exp (-\mu T))
$$

and the probability of having $N$ bidders at time $T$ is given by

$$
q_{N}(T)=e^{-\tilde{\lambda}(T)} \frac{\tilde{\lambda}(T)^{N}}{N !}
$$

Thus, the expected number of bidders in the auction at time $T$ is given by $\tilde{\lambda}(T)$. Since for all $\mu>0$,

$$
\frac{\partial \tilde{\lambda}(T)}{\partial T}=\lambda e^{-\mu T}>0
$$

and

$$
\frac{\partial^{2} \tilde{\lambda}(T)}{\partial T^{2}}=-\lambda \mu e^{-\mu T}<0
$$

the expected number of bidders participating in the auction is an increasing but concave function of the auction's closing time. This effect arises because some of the bidders find an alternative and drop out from the auction. As will be shown shortly, it is this phenomenon which potentially makes the auction inferior to the store as a selling institution and explains the dominance of the latter in practice. Note that if bidders do not have outside options $(\mu \rightarrow 0)$, $\tilde{\lambda}(T) \rightarrow \lambda T$.

Note also that for all $T>0$,

$$
\frac{\partial \tilde{\lambda}(T)}{\partial \mu}=\frac{\lambda}{\mu^{2}} e^{-\mu T}\left(1+\mu T-e^{\mu T}\right)<0
$$

and

$$
\frac{\partial \tilde{\lambda}(T)}{\partial \lambda}=\frac{1}{\mu}(1-\exp (-\mu T))>0
$$

\footnotetext{
${ }^{9}$ At the time this decision is made, the actual price at which the auction will clear is unknown, so the consumer must implicitly solve the stochastic problem and calculate the expected utility to waiting for the auction.
} 
Thus, the higher the drop out rate, the lower the expected number of bidders participating in the auction at any given time $T$ and the higher the arrival rate, the greater the expected number of bidders. Furthermore, $\lim _{T \rightarrow \infty} \tilde{\lambda}(T)=\lambda / \mu$, so that the number of bidders as time goes to infinity is just the potential bidder arrival rate divided by the departure rate among actual bidders.

The seller's expected payoff from auctioning the good at time $T, \Pi_{A}(T)$, is equal to the present value of the expectation over $N$ of the revenue from an auction with $N$ bidders, $\pi_{N}$ :

$$
\Pi_{A}(T)=e^{-\alpha T} E\left(\pi_{N} \mid \tilde{\lambda}(T)\right)
$$

where $\alpha$ again denotes the discount rate. The seller's problem is to select an auctioning time $T$ and a reservation price $p$ so as to maximize this expression.

Wang (1993) shows that the expected revenue from the auction conditional on receiving $N$ bids is given by

$$
\begin{aligned}
\pi_{N} & =\int_{p}^{\bar{v}} N F^{N-1}(y)(y f(y)-(1-F(y))) d y+F^{N}(p) R \\
& =R+\int_{p}^{\bar{v}} N F^{N-1}(y)(J(y)-R) f(y) d y
\end{aligned}
$$

where $J(y) \equiv y-\frac{1-F(y)}{f(y)}$ denotes the Bulow and Roberts (1989) marginal revenue function. Equation (13) is the standard result that the expected utility from an auction is equal to the expectation of the maximum of the highest bidder's marginal revenue and reservation utility (see Bulow and Klemperer (1996)). Note that because it is the expectation of the second highest order statistic of bidders' valuations, $\pi_{N}$ is an increasing and concave function of $N$.

Taking the first-order condition on (13), the optimal reservation price $p^{*}$ solves $J\left(p^{*}\right)=R$, or, using the definition of $J$,

$$
p^{*}=R+\frac{1-F\left(p^{*}\right)}{f\left(p^{*}\right)}
$$

Note that consistent with the Riley and Samuelson (1981) result, the optimal reservation price $p^{*}$ does not depend on the number of bidders participating in the auction and is identical to the store's posted price.

Letting $\pi(\tilde{\lambda}(T)) \equiv E\left(\pi_{N} \mid \tilde{\lambda}(T)\right)$, the seller's expected utility from an auction taking place at time $T$ can be written as

$$
\Pi_{A}(T)=e^{-\alpha T} \pi(\tilde{\lambda}(T))=e^{-\alpha T} \sum_{N=0}^{\infty} e^{-\tilde{\lambda}(T)} \frac{\tilde{\lambda}(T)^{N}}{N !} \pi_{N}
$$


Using the expression for $\pi_{N}$, and as shown in Wang (1993), one can rewrite $\pi(\tilde{\lambda}(T))$ in an alternate form convenient to derive some of the results below, namely

$$
\begin{aligned}
\pi(\tilde{\lambda}(T)) & =\sum_{N=0}^{\infty} e^{-\tilde{\lambda}(T)} \frac{\tilde{\lambda}(T)^{N}}{N !}\left(\int_{p}^{\bar{v}} N F^{N-1}(y)(J(y)-R) f(y) d y+R\right) \\
& =R+\tilde{\lambda}(T) \int_{p}^{\bar{v}} e^{-\tilde{\lambda}(T)(1-F(y))}(J(y)-R) f(y) d y
\end{aligned}
$$

Another helpful result for the subsequent analysis is that the properties of $\pi_{N}$, which is an increasing and concave function of $N$, carry over to $\pi(\tilde{\lambda})$, and therefore to the auction's expected payoff.

Proposition 2: $\pi(\tilde{\lambda})=E\left(\pi_{N} \mid \tilde{\lambda}\right)$ is increasing and concave in $\tilde{\lambda}$.

The larger the number of actual bidders at time $T$, the higher are profits. But profits increase at a decreasing rate in the number of bidders because the second highest order statistic is an increasing, but concave function of the number of observations in a sample, in this case, the number of bidders.

Note that since $\tilde{\lambda}(T)$ is strictly increasing and concave in $T$, proposition 2 implies that the expected (undiscounted) revenue from the auction, $\pi(\tilde{\lambda}(T))$, will be strictly increasing and concave in the auction's closing time $T$ as well:

$$
\begin{gathered}
\frac{\partial \pi}{\partial T}=\frac{\partial \pi}{\partial \tilde{\lambda}} \frac{\partial \tilde{\lambda}}{\partial T}>0 \\
\frac{\partial^{2} \pi}{\partial T^{2}}=\frac{\partial^{2} \pi}{\partial \tilde{\lambda}^{2}}\left(\frac{\partial \tilde{\lambda}}{\partial T}\right)^{2}+\frac{\partial \pi}{\partial \tilde{\lambda}} \frac{\partial^{2} \tilde{\lambda}}{\partial T^{2}}<0
\end{gathered}
$$

This property again follows from the nature of $\pi_{N}$ as the expectation of the second highest order statistic of bidders' valuations.

\subsection{Properties of the Optimal Auction}

At this point, a slight digression is useful. We consider the properties of an optimal auction and how they vary with the discount rate, the arrival rate of bidders, and the availability of alternatives. The purpose is not so much to characterize auctions, but rather as a foundation for understanding why retail stores dominate even the best auction under a large variety of circumstances. 
Choosing the auction's optimal closing time $T^{*}$ involves a tradeoff for the seller: on the one hand, a higher $T$ increases the expected number of bidders participating in the auction, $\tilde{\lambda}(T)$ and therefore the expected revenue from the sale $\pi(\tilde{\lambda}(T))$. On the other hand, as the closing time $T$ is increased, discounting reduces the present value of the proceeds. The optimal closing time $T^{*}$ is the one that balances these two effects.

As in the case of the store, if the discount rate is very high, the seller will decide to consume the good himself rather than trying to auction it. In the context of the auction, this translates to an optimal closing time of 0 . More specifically, one can establish the following result:

Proposition 3: If the discount rate exceeds $\alpha^{*} \equiv \lambda \frac{\int_{p}^{\bar{v}}(J(y)-R) f(y) d y}{R}$, then the seller prefers not to auction the good.

The economic intuition behind proposition 3 is the following: if the discount rate lies above the instantaneous proportional gain from auctioning the good (which is equal to the product of the arrival rate $\lambda$ and the proportional premium of expected marginal utility over the reservation price, $\left.\frac{\int_{p}^{\bar{v}}(J(y)-R) f(y) d y}{R}\right)$, it is not worth running an auction, and the seller prefers consuming the good himself (trivially, a sale is again always optimal if $R=0$ ). If the discount rate lies below $\alpha^{*}$, then it is worth for the seller to wait some positive time $T$ before closing the auction, hoping that at least one bidder will show up. Note also that as the rate of bidder arrivals $\lambda$ increases, so does the critical value $\alpha^{*}$ and the seller is more likely to attempt a sale.

It is worth noting that the critical values $\alpha^{*}$ in propositions 1 and 3 are in indeed equivalent, since

$$
\begin{aligned}
\lambda \frac{\int_{p^{*}}^{\bar{v}}\left(y-\frac{1-F(y)}{f(y)}-R\right) f(y) d y}{R} & =\lambda \frac{\int_{p}^{\bar{v}}(y-R) f(y) d y-(\bar{v}-p)+\left.y F(y)\right|_{p} ^{\bar{v}}-\int_{p}^{\bar{v}} y f(y) d y}{R} \\
& =\lambda \frac{\left(p^{*}-R\right)\left(1-F\left(p^{*}\right)\right)}{R}
\end{aligned}
$$

Thus, whenever $\alpha \geq \alpha^{*}$, there is no attempt to sell the good, and neither store nor auction are run. In what follows, we therefore assume that $\alpha<\alpha^{*}$, so that the seller does attempt a sale and the question of which structure is preferable (i.e., store or auction) becomes relevant. We now show that if attempting a sale is optimal, the auction has an unique optimal closing time $T^{*}$ :

Proposition 4: Whenever $0<\alpha<\alpha^{*}$, there exists a unique $T^{*}>0$ maximizing the auction's expected proceeds $\Pi_{A}(T)$. 
The results in propositions 3 and 4 arise because of the tradeoff between waiting for more bidders to show up (which increases the expected revenue from the auction at time $T$ ) and the effect of discounting (which reduces its present value). Note that in the special case in which $\alpha \rightarrow 0$, it is optimal for the seller to wait a very long time before closing the auction. Provided that $\lambda \gg \mu$, expected bidder participation in the optimal auction will be very high, and $\Pi_{A} \rightarrow \bar{v}$.

The next result demonstrates that changes in the discount rate $\alpha$, the rate of bidder arrivals $\lambda$ and the availability of outside options $\mu$ have an unambiguous impact on the proceeds of the auction.

Proposition 5: $\Pi_{A}\left(T^{*}\right)$ is strictly decreasing in the discount rate $\alpha$ and the availability of outside options $\mu$ and strictly increasing in market thickness $\lambda$.

The next three results characterize the properties of the optimal auction as a function of the discount rate $\alpha$, the rate of bidder arrivals $\lambda$ and the availability of outside options $\mu$. When varying $\lambda$ and $\mu$, the optimal expected number of bidders chosen by the seller, $\tilde{\lambda}\left(T^{*}\right)$ is a more appropriate description of the seller's auctioning strategy than the auction's closing time $T^{*}$, which by itself does not say much about how the auction will look like in terms of bidder participation. Rather than the auction's optimal closing time $T^{*}$, the following results therefore consider the effect of changes in $\alpha, \lambda$ and $\mu$ on the optimal $\tilde{\lambda}\left(T^{*}\right)$.

Proposition 6: The expected number of bidders in the optimal auction, $\tilde{\lambda}\left(T^{*}\right)$, is decreasing in the discount rate $\alpha$, increasing in the bidder arrival rate $\lambda$, and decreasing in the rate of arrival of outside options $\mu$.

The results on the properties of the optimal auction in proposition 6 make intuitive sense. When the discount rate $\alpha$ is low, so that waiting for more bidders is not too costly, the seller increases the expected number of bidders in the optimal auction, $\tilde{\lambda}$. Similarly, if the arrival rate $\lambda$ is high, so that the market is thick, then waiting for one additional bidder entails a lower cost, and the seller increases the expected number of bidders in the optimal auction. Finally, if $\mu$ is high, so that bidders' waiting costs are high or alternatives for the good are easy to find, it is more costly to accumulate bidders and the expected number of bidders in the optimal auction is reduced.

There is an important difference between the impact of the discount rate $\alpha$ and that of the arrival and departure rates $\lambda$ and $\mu$. In the case of changes in the discount rate, there is a 
monotonic relationship between the expected number of bidders $\tilde{\lambda}$ and the auction's optimal closing time $T^{*}$. Waiting longer or waiting for more bidders amount to the same thing, and the auction's optimal closing time is unambiguously decreasing in $\alpha$. On the other hand, in the case of $\lambda$ and $\mu$, proposition 6 allows to make predictions about the expected number of bidders participating in the auction but not about the auction's closing time $T$. Closing time and number of bidders can move in opposite directions when these parameters are varied, as the following numerical example illustrates. Suppose that bidders' valuations are uniformly distributed on the interval $[0,1]$. Then, the reservation price is given by $p^{*}=(1+R) / 2$, and one has

$$
\pi(\tilde{\lambda})=R+\int_{\frac{1+R}{2}}^{1} e^{-\tilde{\lambda}(1-y)} 2\left(y-\frac{1+R}{2}\right) d y=1-2 \frac{1-e^{-\tilde{\lambda} \frac{1-R}{2}}}{\tilde{\lambda}}
$$

Therefore, using the fact that $T=-\ln (1-\tilde{\lambda} \mu / \lambda) / \mu$, the expected profit from the auction can be expressed as a function of $\tilde{\lambda}$ as

$$
\Pi_{A}(\tilde{\lambda})=\left(1-\tilde{\lambda} \frac{\mu}{\lambda}\right)^{\alpha / \mu}\left(1-2 \frac{1-e^{-\tilde{\lambda} \frac{1-R}{2}}}{\tilde{\lambda}}\right)
$$

Figure 1 depicts the optimal expected number of bidders in the auction $\tilde{\lambda}\left(T^{*}\right)$ (upper panel) and the corresponding closing time $T^{*}$ (lower panel) as a function of the arrival rate $\lambda$. Note that as the arrival rate increases, so does the optimal expected number of bidders. However, the optimal length of the auction $T^{*}$ falls. Intuitively, when the arrival rate is high, it is less costly to acquire additional bidders. The seller takes some of the reduced cost in the form of more bidders, and some in the form of a lower $T^{*}$, which has value to him because of a positive discount rate.

The analysis in this section implies that whenever $\alpha$ or $\mu$ are high or $\lambda$ is low, the expected number of bidders in the auction $\tilde{\lambda}$ will be low. But then, the probability of having more than one bidder show up in the auction will be low as well, and the auction will look more like a store in the limit. Intuitively, one would therefore expect the store to dominate the auction for high $\alpha$ and $\mu$ and for low $\lambda .^{10}$ The next section shows that this is indeed the case.

\footnotetext{
${ }^{10}$ It is worth noting that given the equivalence in proposition 2 , this result would follow immediately if an auction closing after a given number of bids $N$ have been received had been used in the analysis instead of an auction closing at a fixed time $T$. In this case, the store is just the corner case of an auction in which the number of bids before clearing is 1 .
} 

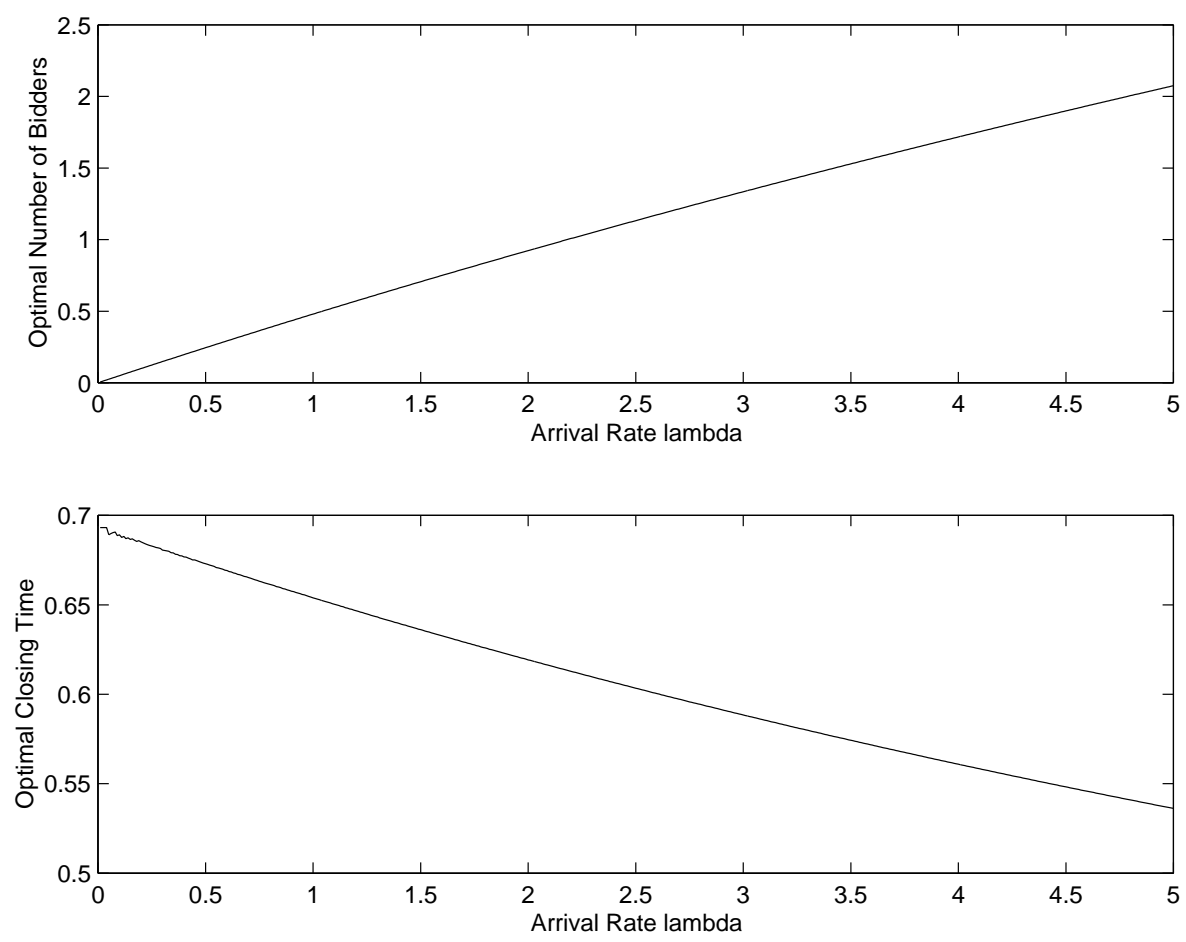

Figure 1: Optimal expected number of bidders in the auction $\tilde{\lambda}\left(T^{*}\right)$ and the corresponding closing time $T^{*}$ (value of the parameters: $\alpha=1$, $R=0, \mu=1)$.

\section{The Optimal Market Structure}

We now turn to the question of which market structure - store or auction - will be optimal for the seller. We first present a general condition under which the auction can dominate the store. In a second step, we show how the optimality of the store or the auction depends on the seller's time preference and the extent to which the good is perishable $(\alpha)$, the thickness of the market $(\lambda)$ and the extent of waiting costs and availability of outside options $(\mu)$.

Proposition 7: The auction is the preferred structure if and only if there exists some $\tilde{\lambda} \in] 0, \lambda / \mu[($ for $\mu \neq 0$ ) or some $\tilde{\lambda}>0$ (for $\mu=0$ ) such that

$$
\begin{array}{rr}
\left(1-\tilde{\lambda} \frac{\mu}{\lambda}\right)^{\alpha / \mu} \pi(\tilde{\lambda})-\frac{\lambda}{\alpha+\lambda}\left(p^{*}\left(1-F\left(p^{*}\right)\right)+R F\left(p^{*}\right)\right)>0 & (\mu \neq 0) \\
e^{-\alpha \tilde{\lambda} / \lambda} \pi(\tilde{\lambda})-\frac{\lambda}{\alpha+\lambda}\left(p^{*}\left(1-F\left(p^{*}\right)\right)+R F\left(p^{*}\right)\right)>0 & (\mu=0)
\end{array}
$$

The intuition for proposition 7 is the following. The store has the advantage that no waiting is necessary between the time that a good is spotted and consumed. It also has the advantage 
that once the good is discovered, it can be consumed with certainty as long as the seller's demand price is paid. In order for an auction to dominate a store, other factors must offset these advantages. The conditions are first that a sufficient number of bidders $\tilde{\lambda}$ must be able to accumulate in order for $\pi(\tilde{\lambda})$ to rise significantly above the expected payoff from the store. The maximum achievable expected number of bidders in the auction, $\lim _{T \rightarrow \infty} \tilde{\lambda}=\lambda / \mu$, depends positively on market thickness $\lambda$ and negatively on the drop-out rate $\mu$. Second, this accumulation must occur sufficiently quickly so that the payoff from the auction does not get discounted too heavily. As is apparent from the expressions $T=-\ln (1-\tilde{\lambda} \mu / \lambda) / \mu$, this accumulation will occur faster, the higher $\lambda$ and the lower $\mu$. Thus, fast arrivals and slow defections positively affect both factors required for the auction to dominate the store.

Waiting until more potential consumers arrive has social advantages because the good is more likely to get into the "right" hands, i.e., to the consumer in society who attaches the highest value to the good. The disadvantage is that all prior bidders and the seller must wait for additional time to pass, which imposes a real cost. Of course, the value of getting the good into the right hands will be higher the more dispersed buyers' valuations.

Similar tradeoffs between the price obtained and the relative costs of running stores and auctions underly many of the results in the existing literature. For example, De Vany (1987) compares stores, time-based auctions (which close after a fixed time has elapsed) and thicknessbased auctions (which close once a certain number of bids has been received) from the perspective of transactions costs. He shows that the higher expected selling price in both auction forms can be offset by their higher transactions costs, which, in his model, are captured by the seller's holding cost and the buyers' inspection and waiting cost. ${ }^{11}$ As a result of these higher transactions costs, the store tends to dominate the two auctioning schemes when the distribution of buyer valuations is not too diffuse, while the auction tends to be the preferred structure when this dispersion is high. Wang (1993) focuses on the explicit costs of running stores and auctions. He shows that the advantage of running auctions periodically instead of selling goods in stores arises when the storage costs incurred by the auction are lower than the displaying costs incurred by a store, which is his working assumption. He also establishes the result that auctions tend to dominate stores when the dispersion of buyers' valuations is high. ${ }^{12}$

By contrast, our focus is on the tradeoff between a higher expected selling price and waiting costs. Waiting costs affect the seller through two channels: perishability and bidder defections. Faster arrivals and slower departures reduce the impact of each and thus tend to favor the

\footnotetext{
${ }^{11}$ Although De Vany computes bidders' waiting cost explicitly in his analysis, he does not allow bidders facing high waiting costs to search for alternatives and defect from the auction.

${ }^{12}$ Wang (1998) extends some of these results to a correlated private values setting.
} 

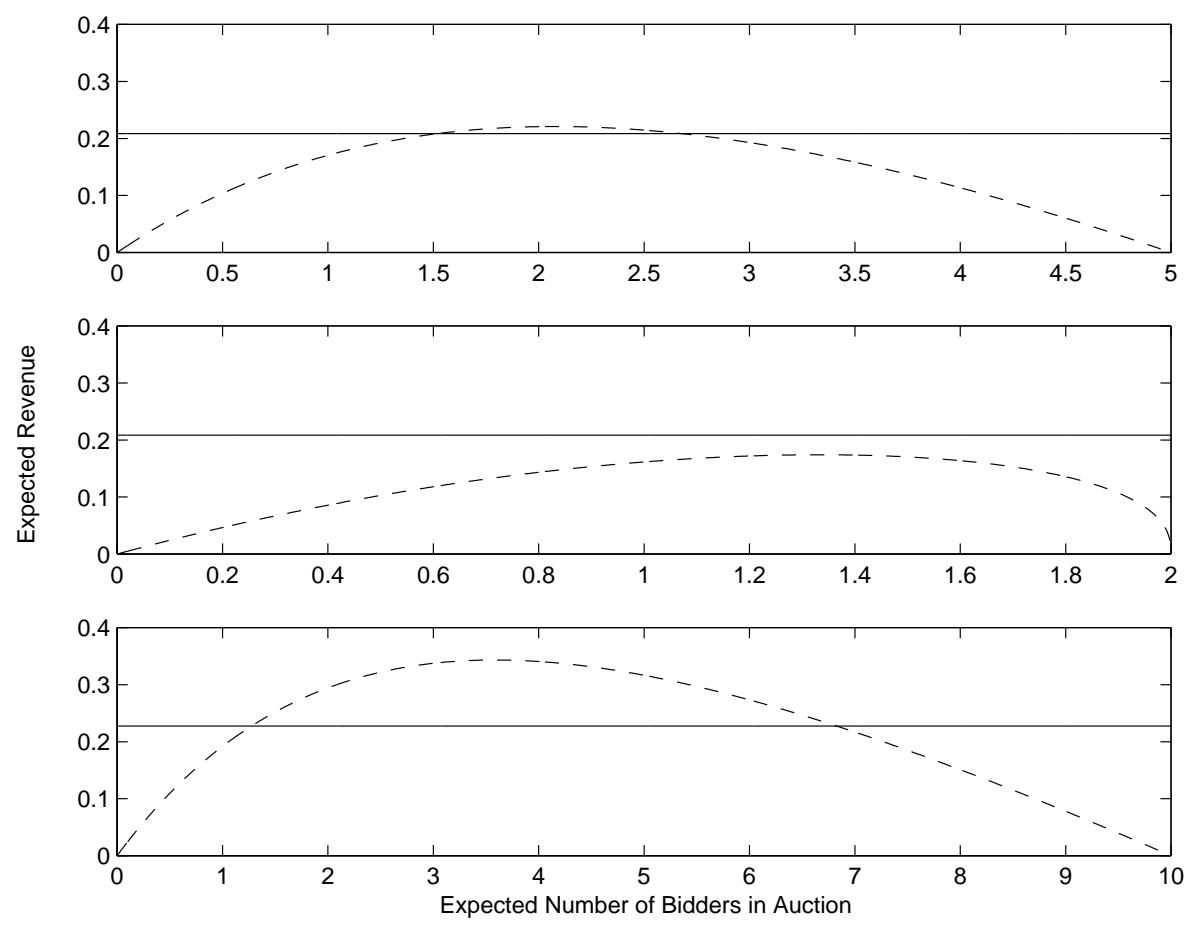

Figure 2: The choice between store (solid line) and auction (dotted line) depending on market thickness $\lambda$ and waiting costs $\mu$ (value of the parameters: $\alpha=1, R=0,\{\lambda, \mu\} \in\{\{5,1\},\{5,2.5\},\{10,1\}\})$.

auction. This can be illustrated using a numerical example. Suppose again that bidders' valuations are uniformly distributed on the interval $[0,1]$. Then, using (21) and the fact that the optimal price for the store is the same as for the auction, $p^{*}=(1+R) / 2$, the condition for the auction to dominate is that there exists a $\tilde{\lambda}$ such that

$$
\left(1-\tilde{\lambda} \frac{\mu}{\lambda}\right)^{\alpha / \mu}\left(1-2 \frac{1-e^{-\tilde{\lambda} \frac{1-R}{2}}}{\tilde{\lambda}}\right)-\frac{\lambda}{\alpha+\lambda}\left(\frac{1+R}{2}\right)^{2}>0
$$

The expected revenue from the auction as a function of $\tilde{\lambda}$ and the expected revenue of the store are depicted in Figure 2 for three different situations. In the base case with $\lambda=5$ and $\mu=1$ (top panel), the auction (dotted line) slightly dominates the store (solid line) if a $\tilde{\lambda}$ around 2 is selected. If the drop-out rate $\mu$ is increased to 2.5 (middle panel), then the auction no longer dominates the store: no $\tilde{\lambda}$ can be found such that (22) is satisfied. Finally, if the arrival rate $\lambda$ is increased to 10 (lower panel), the auction dominates the store more clearly than in the base case.

This example suggests that a high market thickness $\lambda$ and low availability of substitutes $\mu$ should make dominance of the auction more likely. The next two propositions show that this intuition is correct. 
Proposition 8: For all parameter constellations $(\alpha, \mu)$ such that selling is optimal $\left(\alpha<\alpha^{*}\right)$, there exists a rate of bidder arrivals $\lambda_{0}$ such that auctioning is optimal if $\lambda>\lambda_{0}$.

The intuition for proposition 8 is clear. What matters for the seller's choice is the cost of waiting for additional bidders. When the rate of bidder arrivals is high, this cost is low because many bidders will accumulate even if the auction's closing time is set early. Thus, the seller decides to run an auction rather than a store to take advantage of the higher expected selling price in the auction. ${ }^{13}$ An interesting implication of proposition 8 is that the optimal way to sell the same good, with the same distribution of bidder valuations and the same perishability $\alpha$, can be either a store or an auction depending on the thickness of the market.

Anecdotal empirical evidence is completely in line with this analysis. For example, the market for fresh fish and the Amsterdam fresh flower market, which are characterized by high levels of thickness (with all interested bidders showing up every morning at the announced time to bid), are typically run using auctions. At the retail level, where the rate of arrivals is low, however, those same goods are sold using posted prices. ${ }^{14}$ The real estate market provides another instructive example. Houses are most commonly sold using posted prices. When the market is very thick, however, auctions are commonly used. Such a switch from posted prices to auctions has been observed in the California real-estate market in the late 1990's.

Turning to the impact of bidders' waiting cost and false trading, the next result shows that if the drop-out rate $\mu$ is high, the seller will always prefer a store.

Proposition 9: For all parameter constellations $(\alpha, \lambda)$ such that selling is optimal $\left(\alpha<\alpha^{*}\right)$, there exists a drop out rate $\mu_{0}$ such that the seller always prefers the store to the auction if $\mu>\mu_{0}$.

\footnotetext{
${ }^{13}$ Of course, the cost of obtaining additional offers from buyers can arise from sources other than the good's perishability and previous arrivals' defections. Riley and Zeckhauser (1983) and McAfee and McMillan (1988) consider the effect of communication costs in settings in which the seller must bear a fixed cost for each new buyer that he contacts. Riley and Zeckauser (1983) demonstrate that relative to any haggling strategy, a posted price maximizes seller profits. McAfee and McMillan (1988) show that the auction is optimal when the communication cost is zero. When the communication cost is nonzero, the seller first approaches potential buyers sequentially. If he exhausts the entire set of potential buyers, he sells to the buyer with the highest valuation. In the limiting case of infinitely many potential buyers, the optimal mechanism is a posted price. Arnold and Lippman (1995) consider the role of the number of units up for sale and demonstrate that auctions are preferred when the number of items is large, and posted prices when it is small.

${ }^{14}$ It is true, however, that the posted price is not the typical selling price. But the feature that is crucial for this analysis is that there is no time in the future until which a potential buyer is forced to wait before the sale is actualized.
} 
The result in Proposition 9 demonstrates that the speed at which bidders find an alternative plays a critical role in the choice between store and auction. The higher bidders' waiting costs and the easier alternatives can be found, the more likely a store will be used. ${ }^{15}$ Again, this result is completely in line with what one observes in practice. For instance, groceries, for which waiting costs are high (since immediacy is important to buyers) and alternatives very easy to find, are sold using stores. Rembrandt paintings, for which immediacy is not important to buyers and alternatives are very difficult to find, are usually sold by auction, while artwork of less famous artists, for which alternatives are easier to find, is exposed in art galleries and sold by what is essentially a posted price. Paintings by the same artist are sold using one or the other structure at different stages in his career. Indeed, at the beginning of his career, Picasso's paintings were commonly sold in art galleries, but are nowadays most commonly sold by auction.

Much the same observation applies to antiques: most items are sold at fixed price, but truly unique items for which substitutes are difficult to find are sold by auction. Luxury goods, such as jewels and gold watches, provide another illustration along similar lines. Most are sold at a fixed price because similar items can be found elsewhere. Truly unique pieces for which substitutes are very difficult to find, such as Faberge eggs and antique watches, are usually sold by auction.

Commodities provide another interesting application of this analysis. Cash-settled commodity futures contracts, which are homogeneous, are usually traded on centralized exchanges using double auctions, which are essentially posted prices, since trades are executed as soon as a bid or offer is accepted. ${ }^{16}$ On the other hand, commodities for physical delivery at a certain place and day, for which substitutes are difficult to find, are usually sold by auction among the different potential buyers present at a given location. Indeed, the availability of substitutes plays such an important role in physical commodities trading that traders commonly maintain maps locating the different ships containing a given commodity on the walls of their office. Note that as in the case of the arrival rate, two goods with identical distributions of bidder valuations can be sold optimally either using a store or an auction, depending on the size of waiting costs and whether alternative purchase opportunities are available to the bidders or not.

Much of this can be put in familiar terms of elasticity. The elasticity of demand is high when

\footnotetext{
${ }^{15} \mathrm{~A}$ related result is obtained by Ehrman and Peters (1993). Considering a setting in which buyers have a certain alternative that yields them a sure surplus, they show that the auction dominates when this surplus is zero. When this surplus is high, a posted price-like scheme will be preferred by the seller.

${ }^{16}$ See De Vany (1987) for a more detailed discussion of the fact that double auctions are closer to posted prices than to auctions.
} 
good substitutes are available. The availability of good substitutes depends on low search costs and on the existence of other products that can do approximately the same thing as the one in question. The elasticity of supply being high means that there are many identical goods available at close to the same price. In the example of non-perishables that are produced by many firms in an industry with free entry, it is difficult to imagine that auctions could survive. With low search costs, no seller could receive more than marginal cost because consumers would always have a ready alternative, namely, buying the same good from another seller. Under such circumstances, the supply to any given buyer would be perfectly elastic at the market price, which can be thought of as having an infinite rate of arrival of alternatives. ${ }^{17}$

New goods have higher supply elasticities than used ones. In an extreme case, the supply of a "used" good, such as a particular Picasso painting is completely inelastic and good substitutes are difficult to find. New goods, such as a particular digital camera, are supplied more elastically. Auctions are more common for used goods than they are for new ones. This is true even in electronic markets like eBay. Used items or those inelastically supplied such as rare antiques, are sold primarily by auctions, whereas new ones, such as a particular book that can be produced at almost constant marginal cost, are sold through stores.

Proposition 9 does imply that a higher drop out rate $\mu$ makes the use of posted prices more likely, but it does not allow a precise characterization of when an auction or a store will be preferred. The next proposition puts a much tighter bound on the parameter constellations under which auctions can arise as optimal selling institutions.

Proposition 10: Let $g(\tilde{\lambda}) \equiv \frac{\int_{p^{*}}^{\bar{v}} e^{-\tilde{\lambda}(1-F(y))}(J(y)-R) f(y) d y}{\int_{p^{*}}^{\bar{v}}(J(y)-R) f(y) d y}<1$. An auction such that $\tilde{\lambda} g(\tilde{\lambda})<$ $\frac{\lambda}{\alpha+\lambda}-\frac{\alpha}{\alpha+\lambda} \frac{R}{\left(p^{*}-R\right)\left(1-F\left(p^{*}\right)\right)}$ cannot dominate a store.

Proposition 10 implies that for the auction to be able to dominate the store, a minimum expected number of bidders $\tilde{\lambda}$ is required in the auction. This is because the benefit of using an auction as opposed to a store stems from the fact that a price higher than the reservation

\footnotetext{
${ }^{17}$ Although our analysis is cast in terms of availability of outside alternatives, similar effects arise when a monopoly seller faces capacity constraints in production. Harris and Raviv (1981) demonstrate that when the firm can produce as many units of output as desired at constant cost, the optimal selling mechanism is a fixed price, whereas an auction with a reserve price will be preferred if the capacity constraint is binding (i.e., if the number of potential buyers exceeds the capacity constraint). Along similar lines, Milgrom (1989) notes that posted prices are commonly used for standardized, inexpensive items sold in stores and for which all comers can be served, when it is too expensive to gather the competing buyers together or if the timing of buyer demands varies. On the other hand, when goods are not standardized or when the market clearing prices are highly unstable, auctions are preferred.
} 

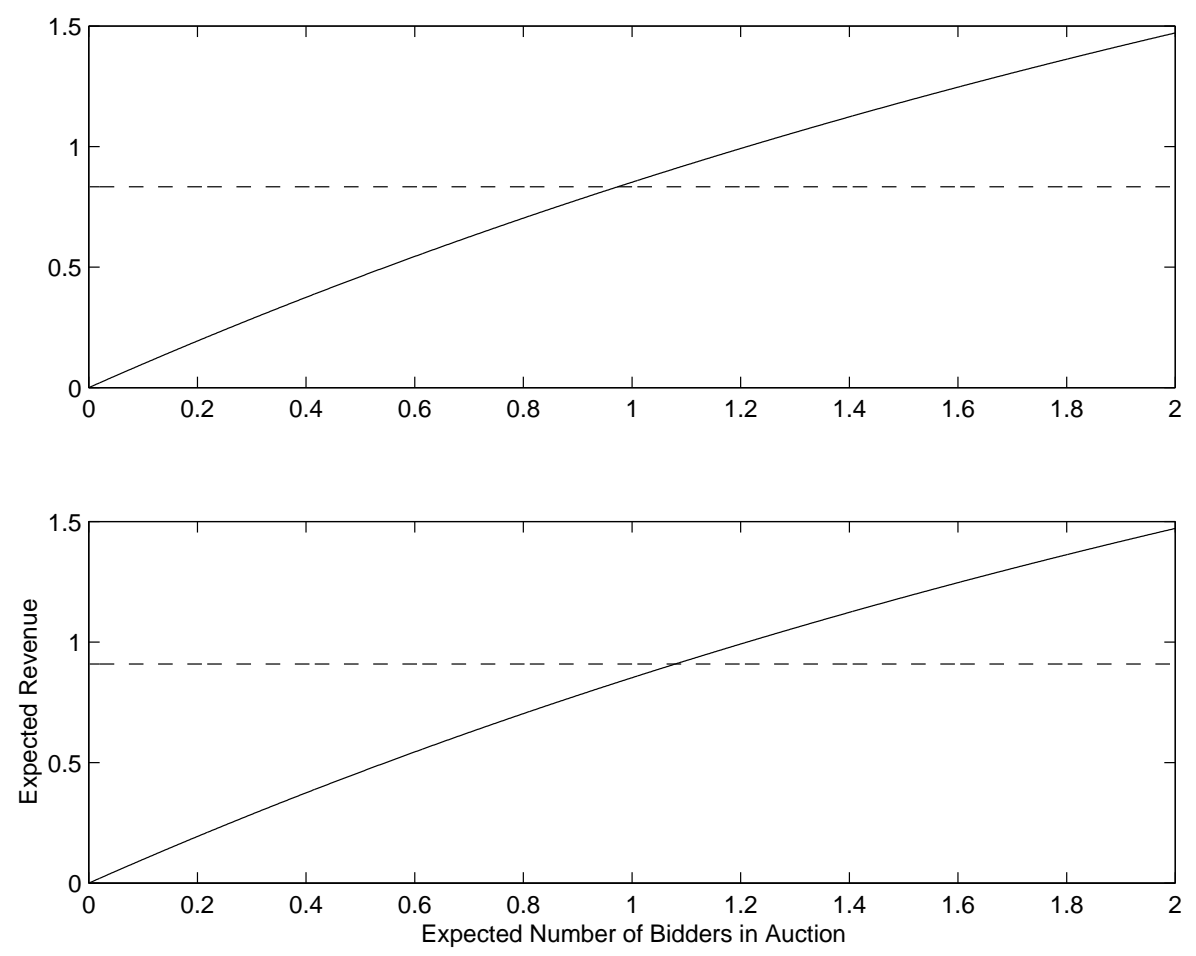

Figure 3: Minimum value of $\tilde{\lambda}$ below which the store is always preferred to the auction (value of the parameters: $\alpha=1, R=0, \lambda \in\{5,10\}$ ).

price $p^{*}$ can be extracted. This, however, can only occur if the average number of bidders is sufficiently high.

Proposition 10 is best illustrated using a numerical example. Suppose again that that bidders' valuations are uniformly distributed on the interval $[0,1]$. Using the fact that $p^{*}=$ $(1+R) / 2$, one has

$$
g(\tilde{\lambda})=\frac{\int_{\frac{1+R}{2}}^{1} e^{-\tilde{\lambda}(1-y)}\left(y-\frac{1+R}{2}\right) d y}{\int_{\frac{1+R}{2}}^{1}\left(y-\frac{1+R}{2}\right) d y}=\frac{\frac{1}{\tilde{\lambda}^{2}}\left(e^{-\tilde{\lambda} \frac{1-R}{2}}-1\right)+\frac{1}{\tilde{\lambda}}\left(\frac{1-R}{2}\right)}{\frac{1}{2}\left(\frac{1-R}{2}\right)^{2}}
$$

Therefore, the store is guaranteed to dominate the auction for all $\tilde{\lambda}$ such that

$$
\frac{\frac{1}{\tilde{\lambda}}\left(e^{-\tilde{\lambda} \frac{1-R}{2}}-1\right)+\frac{1-R}{2}}{\frac{1}{2}\left(\frac{1-R}{2}\right)^{2}}<\frac{\lambda}{\alpha+\lambda}-\frac{\alpha}{\alpha+\lambda} \frac{R}{\left(\frac{1-R}{2}\right)^{2}}
$$

Both $\tilde{\lambda} g(\tilde{\lambda})$ and $\frac{\lambda}{\alpha+\lambda}-\frac{\alpha}{\alpha+\lambda} \frac{R}{\left(\frac{1-R}{2}\right)^{2}}$ are depicted in Figure 3 for $\lambda=5$ and $\lambda=10$. Note that the minimum expected number of bidders in the auction $\tilde{\lambda}$ below which the store will be the preferred structure becomes larger, the larger the arrival rate $\lambda$. This is because the store becomes more profitable as $\lambda$ is increased. 
The result of proposition 10 is related to proposition 7 but somewhat weaker, as the effect of the time required for bidders to accumulate on the value of the sale is not taken explicitly into account. Nevertheless, it is a very convenient starting point to derive results that allow to rule out the dominance of auctions in many circumstances. The reason is that the rate $\mu$ at which bidders find alternatives constrains the maximum expected number of bidders $\tilde{\lambda}$ that the seller can achieve in an auction not to exceed $\lambda / \mu$. Therefore, knowing that there exists a critical $\tilde{\lambda}$ below which the store will dominate the auction, and since $\tilde{\lambda}$ is a strictly decreasing function of $\mu$, a corresponding value for $\mu$ above which it is certain that the store will be preferred to the auction can be determined:

Proposition 11: Whenever $\mu>\lambda \frac{\frac{\lambda}{\alpha}+1}{\frac{\lambda}{\alpha}-\frac{R}{\left(p^{*}-R\right)\left(1-F\left(p^{*}\right)\right)}}$, the store dominates the auction.

Proposition 11 confirms the basic intuition that running an auction, which requires a large number of bidders to work well, becomes less interesting than the store if bidders are not ready to wait because they can find opportunities to purchase the good elsewhere. It also implies that regardless of the distribution of bidders' valuations, if both the discount rate $\alpha$ and the bidder arrival rate $\lambda$ are reduced proportionally, the value of $\mu$ above which the store is guaranteed to dominate the auction falls by the same proportion.

To understand the basic intuition behind proposition 11, is instructive to consider the limiting case in which the discount rate is very low. In such a setting, ignoring outside options would lead to the recommendation that an auction should be run. However, using the condition in proposition 11 and L'Hospital's rule, a sufficient condition for the store to dominate the auction when $\alpha \rightarrow 0$ is that $\mu>\lambda$. The reason is that if this condition is satisfied, the average time $1 / \mu$ until bidders find an alternative to purchase the good elsewhere is lower than the average bidder interarrival time, $1 / \lambda$. As a result, the probability of having two bidders if an auction is run is very low and the seller prefers to sell the good using a store.

What matters is how the departure rate compares to the arrival rate. Whenever the former exceeds the latter, an auction is unable to achieve the number of bidders required to command a premium over the store and will therefore not be selected by the seller. Again, groceries and artwork from famous artists constitute a case in point. The arrival rate on groceries is much higher than on Rembrandt paintings, but substitutes are so easy to find that the store is preferred. The arrival rate on Rembrandt paintings is very low, but alternatives are so difficult to find that the auction can be used.

The next result shows that if the discount rate is sufficiently low, the auction will be the preferred structure. 
Proposition 12: Suppose that $\lambda \gg \mu$. There exists some $\alpha_{0}>0$ so that the auction dominates the store for $\alpha<\alpha_{0}$.

Note that a value of $\tilde{\lambda}$ strictly (and possibly significantly) above 1 must be achievable for the auction to dominate for low $\alpha$. The reason is that because of the concavity of $\pi(\tilde{\lambda})$, Jensen's inequality implies that $\pi(1)<\pi_{1}$, and the auction cannot dominate the store if $\tilde{\lambda}<1$. As a result, the condition $\lambda>\mu$ would not be sufficient to guarantee that the auction dominates the store, and $\lambda \gg \mu$ is required.

Proposition 12 implies that other things equal, goods that are perishable or become obsolete quickly should be sold using stores rather than auctions, while goods whose value does not fall too quickly through time should be sold using auctions. This prediction is consistent with the empirical evidence. Artwork is hardly perishable and sold by auction, while fresh vegetables are typically sold in stores. Again, it is important to note that it is not the rate of perishability as such that will drive the choice between both forms of sale, but how it compares to the arrival rate. Fresh flowers provide a nice illustration. They are equally perishable at the wholesale and at the retail level. At the retail level, the arrival rate is low, and the store is preferred. At the wholesale level, however, the market is thicker and auctions are preferred.

\section{Summary and Conclusion}

Auctions have the undesirable feature that they impose waiting costs on bidders and give rise to false trading. As a result, the seller will often prefer running a retail store to running an auction. Three factors are shown to play a critical role in the seller's choice between store and auction: (1) the perishability of the good and discounting, (2) the thickness of the market, and (3) the extent of buyers' waiting costs and the availability of alternatives. More specifically, the following can be established:

1. If the seller's time preference, the perishability of the good being sold or the speed at which it becomes obsolete are high, then the store tends to be the preferred structure.

2. If the market is very thick in the sense that many bidders arrive per unit time, the auction tends to be preferred to the store.

3. High bidders' waiting costs and the availability of alternatives to purchase an identical or similar good elsewhere tend to favor the store. 
An important implication of our analysis is that none of these factors alone can explain why a given form of sale is selected in practice. Rather, what is important is their relative magnitude. Perishable items can be sold by auction if the arrival rate is high, while nonperishable items may be sold by auction if the market is thin. In the same way, goods for which alternatives are difficult to find may be sold in stores if the arrival rate is low, and goods for which substitutes are easily available by auction if the arrival rate is high. The immediate consequence is that identical goods may be sold in different ways depending on the market environment. In an environment in which arrivals are frequent and alternatives difficult to find, the good will be sold using an auction. However, the store may be the optimal structure to sell the same good if the arrival rate is low and alternatives are hard to find.

The development of markets on the Internet provides an interesting application of this analysis. At first sight, the Internet would seem to favor auctions because it allows the arrival streams of almost the whole world to be pooled together (at least for goods that can be shipped). On the other hand, Internet technology makes searching for alternatives much easier, which tends to favor stores. This explains why stores have become more and more common on the Internet in the last few years, with even originally all-auction electronic marketplaces such as eBay now allowing sellers to run retail stores rather than auctions.

The Internet actually provides an interesting laboratory to assess the impact of the different factors studied in this paper. As mentioned in Pinker et al. (2002), the Internet has reduced explicit auctioning costs to such an extent that almost anything can now be sold online. ${ }^{18}$ Accordingly, firms have been experimenting auctions and posted prices with various goods. An example is Priceline, which made an abortive attempt to run grocery auctions. By emphasizing the role of immediacy and the availability of substitutes, this paper makes clear why auctions were inappropriate in this setting.

To some extent, the Internet may allow to disentangle the impact of the different factors. Consider for example immediacy and false trading. Internet auctions and stores will both fail if immediacy is very important to buyers. If immediacy is not too important but false trading is, then Internet stores will succeed and Internet auctions will fail. The appearance of "buy now" prices on many Internet auction sites reflects the relative importance of these factors.

\footnotetext{
${ }^{18}$ Lucking-Reiley (2000) finds that auctioneer fees are much lower on the Internet than in traditional auctions. As noted in Pinker et al. (2002), this supports the view that the Internet has made auctioning more accessible.
} 


\section{A Distribution of the Number of Bids at time $T$}

In this appendix, we derive the distribution of the number of bidders participating in the auction at time $T$. To do so, the auction is best viewed as a queuing system in which customers arrive at a rate $\lambda$ and each customer in the system leaves at a rate $\mu$. Let $q_{N}(t)$ denote the probability that there are $N$ bidders in the system at time $t$. These probabilities must satisfy the following system of Chapman-Kolmogorov differential equations:

$$
\begin{aligned}
\frac{d q_{0}(t)}{d t} & =-\lambda q_{0}(t)+\mu q_{1}(t), \quad(N=0) \\
\frac{d q_{N}(t)}{d t} & =-(\lambda+N \mu) q_{N}(t)+(N+1) \mu q_{N+1}(t)+\lambda q_{N-1}(t), \quad(N>0)
\end{aligned}
$$

The intuition behind this system is the following: if the system is currently empty $(N=0)$, there is a probability $\lambda$ per unit time that a bidder will show up, reducing the probability that the system remains empty by $\lambda$. On the other hand, if there is one bidder in the system $(N=1)$, there is a probability $\mu$ per unit time that a bidder will leave it and bring it to state 0 . Together, these factors imply (25). More generally, if there are currently $N$ bidders in the system, there is a probability $\lambda$ per unit time that a bidder will arrive and bring it to state $N+1$, and a probability $N \mu$ that one of the bidders will leave it and bring it to state $N-1$. On the other hand, if the system is in state $N-1$, there is a probability $\lambda$ that one bidder will arrive and bring the system to state $N$. Finally, if there are $N+1$ bidders in the system, there is a probability $(N+1) \mu$ that one of the bidders will depart. This then implies (26).

To solve this system, multiply the equation for $N$ by $z^{N}$ and sum over all $N$ to obtain

$$
\sum_{N=1}^{\infty} z^{N} \frac{d q_{N}(t)}{d t}+\mu(z-1) \sum_{N=1}^{\infty} N z^{N-1} q_{N}(t)=\lambda(z-1) \sum_{N=0}^{\infty} z^{N} q_{N}(t)
$$

Defining $Q(z, t)=\sum_{N=0}^{\infty} z^{N} q_{N}(t)$, this equation can be rewritten as

$$
\frac{\partial Q}{\partial t}+\mu(z-1) \frac{\partial Q}{\partial z}=\lambda(z-1) Q
$$

This equation can then be solved with the initial condition that there are no bidders at time $0, q_{0}(0)=1$, yielding

$$
Q(z, t)=\exp \left(\frac{\lambda}{\mu}(z-1)\left(1-e^{-\mu t}\right)\right)
$$

To determine the state probabilities at time $t, q_{N}(t)$, take a Taylor series expansion of $Q$ around $z=0$, holding $t$ constant. The probability of state $N$ will be proportional to the coefficients of $z^{N}$ in that Taylor series. Noting that

$$
\frac{\partial^{N} Q}{\partial z^{N}}=\exp \left(\frac{\lambda}{\mu}(z-1)\left(1-e^{-\mu t}\right)\right)\left(\frac{\lambda}{\mu}\left(1-e^{-\mu t}\right)\right)^{N}
$$


the state probabilities are given by

$$
q_{N}(t)=\left.\gamma \frac{1}{N !} \frac{\partial^{N} Q}{\partial z^{N}}\right|_{z=0}=\gamma \frac{1}{N !} \exp \left(-\frac{\lambda}{\mu}\left(1-e^{-\mu t}\right)\right)\left(\frac{\lambda}{\mu}\left(1-e^{-\mu t}\right)\right)^{N}
$$

with $\gamma$ a scaling constant ensuring that the probabilities sum to 1 . Noting that

$$
\sum_{N=0}^{\infty} \frac{1}{N !} \exp \left(-\frac{\lambda}{\mu}\left(1-e^{-\mu t}\right)\right)\left(\frac{\lambda}{\mu}\left(1-e^{-\mu t}\right)\right)^{N}=1
$$

one has $\gamma=1$ and the probability of having $N$ bidders at time $t$ is given by

$$
q_{N}(t)=\frac{1}{N !} \exp \left(-\frac{\lambda}{\mu}\left(1-e^{-\mu t}\right)\right)\left(\frac{\lambda}{\mu}\left(1-e^{-\mu t}\right)\right)^{N}
$$

Defining $\tilde{\lambda}(t)=\frac{\lambda}{\mu}\left(1-e^{-\mu t}\right)$, the state probabilities (33) can be recognized as those of a Poisson distribution with parameter $\tilde{\lambda}(t)$,

$$
q_{N}(t)=e^{-\tilde{\lambda}(t)} \frac{\tilde{\lambda}(t)^{N}}{N !}
$$

which is the result used in the text. 


\section{B Proofs of Propositions}

\section{Proof of Proposition 1}

Immediate by solving the condition $\Pi_{S}=\frac{\lambda}{\alpha+\lambda}\left(p^{*}\left(1-F\left(p^{*}\right)\right)+F\left(p^{*}\right) R\right)>R$ for $\alpha$.

\section{Proof of Proposition 2}

To establish that $\pi$ is increasing in $\tilde{\lambda}$, take the first derivative of $\pi$ with respect to $\tilde{\lambda}$ to obtain

$$
\frac{\partial \pi}{\partial \tilde{\lambda}}=e^{-\tilde{\lambda}}\left(\sum_{N=1}^{\infty} \pi_{N} \frac{\tilde{\lambda}^{N-1}}{(N-1) !}-\sum_{N=0}^{\infty} \pi_{N} \frac{\tilde{\lambda}^{N}}{N !}\right)=e^{-\tilde{\lambda}}\left(\sum_{N=1}^{\infty} \pi_{N}\left(\frac{\tilde{\lambda}^{N-1}}{(N-1) !}-\frac{\tilde{\lambda}^{N}}{N !}\right)-\pi_{0}\right)
$$

Grouping terms of like powers of $\tilde{\lambda}$ and remembering that $\pi_{N}$ is increasing in $N$ then yields

$$
\frac{\partial \pi}{\partial \tilde{\lambda}}=e^{-\tilde{\lambda}} \sum_{N=0}^{\infty} \frac{\tilde{\lambda}^{N}}{N !}\left(\pi_{N+1}-\pi_{N}\right)>0
$$

To establish concavity of $\pi(\tilde{\lambda})$, differentiate $\pi$ one more time to obtain

$$
\begin{aligned}
\frac{\partial^{2} \pi}{\partial \tilde{\lambda}^{2}} & =e^{-\tilde{\lambda}}\left(\left(\sum_{N=2}^{\infty}\left(\frac{\tilde{\lambda}^{N-2}}{(N-2) !}-\frac{\tilde{\lambda}^{N-1}}{(N-1) !}\right)-\pi_{1}\right)-\left(\sum_{N=1}^{\infty} \pi_{N}\left(\frac{\tilde{\lambda}^{N-1}}{(N-1) !}-\frac{\tilde{\lambda}^{N}}{N !}\right)-\pi_{0}\right)\right) \\
& =e^{-\tilde{\lambda}}\left(\pi_{0}+(\tilde{\lambda}-2) \pi_{1}+\sum_{N=2}^{\infty} \pi_{N}\left(\frac{\tilde{\lambda}^{N-2}}{(N-2) !}-2 \frac{\tilde{\lambda}^{N-1}}{(N-1) !}+\frac{\tilde{\lambda}^{N}}{N !}\right)\right)
\end{aligned}
$$

Grouping terms of like powers of $\tilde{\lambda}$ and using the concavity of $\pi_{N}$ then yields

$$
\frac{\partial^{2} \pi}{\partial \tilde{\lambda}^{2}}=e^{-\tilde{\lambda}} \sum_{N=0}^{\infty} \frac{\tilde{\lambda}^{N}}{N !}\left(\pi_{N+2}-2 \pi_{N+1}+\pi_{N}\right)<0
$$

\section{Proof of Proposition 3}

To establish that the seller prefers not to auction the good if $\alpha \geq \lambda \frac{\int_{p}^{\bar{v}}(J(y)-R) f(y) d y}{R}$, it suffices to show that if this condition is met, $\partial \Pi_{A} / \partial T \leq 0$ at $T=0$ and is nonincreasing thereafter. Using (15), one has

$$
\frac{\partial \Pi_{A}}{\partial T}=e^{-\alpha T} \frac{\partial \pi}{\partial \tilde{\lambda}} \frac{\partial \tilde{\lambda}}{\partial T}-\alpha e^{-\alpha T} \pi=e^{-\alpha T}\left(\frac{\partial \pi}{\partial \tilde{\lambda}} \frac{\partial \tilde{\lambda}}{\partial T}-\alpha \pi\right)
$$

Using (16) yields

$$
\frac{\partial \pi}{\partial \tilde{\lambda}}=\int_{p}^{\bar{v}} e^{-\tilde{\lambda}(T)(1-F(y))}(J(y)-R)(1-\tilde{\lambda}(T)(1-F(y))) f(y) d y
$$


Noting that $\tilde{\lambda}=0$ for $T=0$, evaluating this expression at $T=0$ yields

$$
\left.\frac{\partial \pi}{\partial \tilde{\lambda}}\right|_{\tilde{\lambda}=0}=\int_{p}^{\bar{v}}(J(y)-R) f(y) d y
$$

Moreover, as $T \rightarrow 0, \partial \tilde{\lambda} / \partial T=\lambda$ and $\Pi_{A}(0)=R$, so the condition for the seller to prefer no sale reads

$$
\left.\frac{\partial \Pi_{A}}{\partial T}\right|_{T=0}=\lambda \int_{p}^{\bar{v}}(J(y)-R) f(y) d y-\alpha R \leq 0
$$

Solving for $\alpha$ then establishes the condition for optimality of no sale. In order to establish that no other $T$ can yield a higher expected revenue, it suffices to show that $\Pi_{A}$ is concave in $T$. Note that

$$
\frac{\partial^{2} \Pi_{A}}{\partial T^{2}}=e^{-\alpha T}\left(\frac{\partial^{2} \pi}{\partial \tilde{\lambda^{2}}} \frac{\partial \tilde{\lambda}}{\partial T}+\frac{\partial \pi}{\partial \tilde{\lambda}}\left(\frac{\partial^{2} \tilde{\lambda}}{\partial T^{2}}-\alpha \frac{\partial \tilde{\lambda}}{\partial T}\right)\right)-\alpha \frac{\partial \Pi_{A}}{\partial T}
$$

Using the concavity of $\pi$ and $\tilde{\lambda}$, the term in parentheses is negative. But this implies that at any point where $\partial \Pi_{A} / \partial T=0, \Pi_{A}$ is concave in $T$, implying that all $T$ such that $\partial \Pi_{A} / \partial T=0$ must be maxima. As $\Pi_{A}$ is (weakly) decreasing at $T=0$, it cannot reach a maximum for some $T>0$ without first reaching a minimum. As there can be no such minima, $\Pi_{A}$ can have no maxima other than $T^{*}=0$ either, implying that $T^{*}=0$ is the unique optimal "selling" strategy when $\alpha \geq \lambda \frac{\int_{p}^{\bar{v}}(J(y)-R) f(y) d y}{R}$.

\section{Proof of Proposition 4}

Recall that whenever $\alpha<\alpha^{*}, \Pi_{A}$ is increasing in $T$ at $T=0$, and that $\Pi_{A}(0)=R>0$. Therefore, the existence of an optimal $T^{*}>0$ can be established by noting that $\pi(\tilde{\lambda}(T))$ is bounded, implying that

$$
\lim _{T \rightarrow \infty} \Pi_{A}(T)=\lim _{T \rightarrow \infty} e^{-\alpha T} \pi(\tilde{\lambda}(T))=0<\Pi_{A}(0)
$$

To establish uniqueness, it suffices to show that $\Pi_{A}$ is concave in $T$ for all $T$ such that $\partial \Pi_{A} / \partial T=0$, a result that was already derived in the proof of proposition 3 .

\section{Proof of Proposition 5}

Note that for all $T>0, \partial \Pi_{A} / \partial \alpha=-T e^{-\alpha T} \pi(\tilde{\lambda})<0, \partial \Pi_{A} / \partial \mu=e^{-\alpha T}(\partial \pi / \partial \tilde{\lambda})(\partial \tilde{\lambda} / \partial \mu)<0$ and $\partial \Pi_{A} / \partial \lambda=e^{-\alpha T}(\partial \pi / \partial \tilde{\lambda})(\partial \tilde{\lambda} / \partial \lambda)>0$. Therefore, the claimed relationships must hold as well for the optimal $T^{*}$. 


\section{Proof of Proposition 6}

\section{A. Discount rate $\alpha$}

Since

$$
\frac{d T^{*}}{d \alpha}=-\left.\frac{\partial^{2} \Pi_{A} / \partial T \partial \alpha}{\partial^{2} \Pi_{A} / \partial T^{2}}\right|_{\frac{\partial \Pi_{A}}{\partial T}=0}
$$

and we have shown in proposition 4 that $\partial^{2} \Pi_{A} / \partial T^{2}<0$ whenever $\partial \Pi_{A} / \partial T=0$, the sign of $d T^{*} / d \alpha$ will be equal to that of $\partial^{2} \Pi_{A} / \partial T \partial \alpha$.

Using (39) yields

$$
\left.\frac{\partial^{2} \Pi_{A}}{\partial T \partial \alpha}\right|_{\frac{\partial \Pi_{A}}{\partial T}=0}=\left.\left(-T e^{-\alpha T}\left(\frac{\partial \pi}{\partial \tilde{\lambda}} \frac{\partial \tilde{\lambda}}{\partial T}-\alpha \pi\right)-e^{-\alpha T} \pi\right)\right|_{\frac{\partial \Pi_{A}}{\partial T}=0}=-e^{-\alpha T} \pi<0
$$

Since $\tilde{\lambda}(T)$ is monotone increasing in $T$, this implies that $d \tilde{\lambda} / d \alpha<0$.

B. Arrival rate $\lambda$

Rewrite the first-order condition (39) as

$$
\frac{\partial \pi / \partial \tilde{\lambda}}{\pi} \frac{\partial \tilde{\lambda}}{\partial T}=\alpha
$$

Next, observe that by concavity of $\pi, \Phi(\tilde{\lambda})=(\partial \pi / \partial \tilde{\lambda}) / \pi$ is a strictly decreasing function of $\tilde{\lambda}$. Using the fact that $\partial \tilde{\lambda} / \partial T=\lambda e^{-\mu T}=\lambda-\tilde{\lambda} \mu,(47)$ can be rewritten as

$$
\Phi(\tilde{\lambda})(\lambda-\tilde{\lambda} \mu)=\alpha
$$

Applying the implicit function theorem to (48) then yields

$$
\frac{d \tilde{\lambda}}{d \lambda}=-\frac{\Phi(\tilde{\lambda})}{\left(\Phi^{\prime}(\tilde{\lambda})(\lambda-\tilde{\lambda} \mu)-\mu \Phi(\tilde{\lambda})\right)}>0
$$

C. Drop-out rate $\mu$

As in the proof for $\lambda$, rewrite the first-order condition as $\Phi(\tilde{\lambda})(\lambda-\tilde{\lambda} \mu)=\alpha$. Applying the implicit function theorem then yields

$$
\frac{d \tilde{\lambda}}{d \mu}=-\frac{-\tilde{\lambda} \Phi(\tilde{\lambda})}{\left(\Phi^{\prime}(\tilde{\lambda})(\lambda-\tilde{\lambda} \mu)-\mu \Phi(\tilde{\lambda})\right)}<0
$$

\section{Proof of Proposition 7}

The condition for the auction to dominate is that

$$
e^{-\alpha T} \pi(\tilde{\lambda})>\frac{\lambda}{\alpha+\lambda}\left(p^{*}\left(1-F\left(p^{*}\right)\right)+R F\left(p^{*}\right)\right)
$$


Using the fact that $\tilde{\lambda}=\frac{\lambda}{\mu}\left(1-e^{-\mu T}\right)$ for $\mu \neq 0$ and $\tilde{\lambda}=\lambda T$ for $\mu=0$, we have $T=-\ln (1-$ $\tilde{\lambda} \mu / \lambda) / \mu$ for $\mu \neq 0$ and $T=\tilde{\lambda} / \lambda$ for $\mu=0$. Thus, $e^{-\alpha T}=(1-\tilde{\lambda} \mu / \lambda)^{\alpha / \mu}$ for $\mu \neq 0$ and $e^{-\alpha \tilde{\lambda} / \lambda}$ for $\mu=0$. Substituting into (51), the result follows.

\section{Proof of Proposition 8}

Auctioning will be optimal if

$$
\Pi_{A}(T)=e^{-\alpha T} \pi(\tilde{\lambda}(T))>\Pi_{S}=\frac{\lambda}{\alpha+\lambda} \pi_{1}
$$

Using the definition of $\pi$, a sufficient condition for the auction to dominate is that

$$
\sum_{N=0}^{\infty} e^{-\tilde{\lambda}(T)} \frac{\tilde{\lambda}(T)^{N}}{N !}\left(\pi_{N}-e^{\alpha T} \pi_{1}\right)>0
$$

or

$$
\sum_{N=2}^{\infty} \frac{\tilde{\lambda}(T)^{N}}{N !}\left(\pi_{N}-e^{\alpha T} \pi_{1}\right)+\tilde{\lambda}(T)\left(1-e^{\alpha T}\right) \pi_{1}+\left(\pi_{0}-e^{\alpha T} \pi_{1}\right)>0
$$

Now, choose $T$ small enough such that $\pi_{2}-e^{\alpha T} \pi_{1}>0$, i.e. $T<\ln \left(\pi_{2} / \pi_{1}\right) / \alpha$ (this assumption is innocuous, as if the auction dominates for that "arbitrarily" chosen $T$, it will necessarily do so for the optimal $T^{*}$ as well), and consider the limit as $\lambda \rightarrow \infty$, which for constant $T$ implies $\tilde{\lambda}(T) \rightarrow \infty$. The summation term contains only positive terms by construction and therefore tends to plus infinity in polynomial progression, whereas the second term tends to minus infinity linearly and the third term is constant. Therefore, for constant $T$, the whole expression tends to plus infinity as $\lambda \rightarrow \infty$, and the auction will be preferred to the store for large enough $\lambda$.

\section{Proof of Proposition 9}

Since $\alpha<\alpha^{*}$, we know that the seller's expected utility from running the store is $\Pi_{S}>R$, so all we need to show is that for $\mu$ high enough, he will achieve at most $R$ using an auction. Note that for all $T, \lim _{\mu \rightarrow \infty} \tilde{\lambda}(T)=0$, so it follows from (16) that for all $T \geq 0, \lim _{\mu \rightarrow \infty} \Pi_{A}(T)=$ $e^{-\alpha T} R \leq R$, and the store is therefore strictly preferred to the auction if $\mu$ is sufficiently high. The uniqueness of the critical $\mu_{0}$ follows from the fact that $\Pi_{A}$ is strictly decreasing in $\mu$ for all $T>0$ while $\Pi_{S}$ does not depend on $\mu$.

\section{Proof of Proposition 10}

The necessary and sufficient condition for the store to dominate is that

$$
e^{-\alpha T}\left(R+\tilde{\lambda}(T) \int_{p^{*}}^{\bar{v}} e^{-\tilde{\lambda}(T)(1-F(y))}(J(y)-R) f(y) d y\right)<\frac{\lambda}{\alpha+\lambda}\left(p^{*}\left(1-F\left(p^{*}\right)\right)+R F\left(p^{*}\right)\right)
$$


Therefore, a sufficient condition for the store to be preferred is that

$$
\tilde{\lambda}(T) \int_{p^{*}}^{\bar{v}} e^{-\tilde{\lambda}(T)(1-F(y))}(J(y)-R) f(y) d y<\frac{\lambda}{\alpha+\lambda}\left(\left(p^{*}-R\right)\left(1-F\left(p^{*}\right)\right)-\alpha R\right)
$$

Using the definition of $g(\tilde{\lambda})$ and the fact that $\int_{p}^{\bar{v}}(J(y)-R) f(y) d y=\left(p^{*}-R\right)\left(1-F\left(p^{*}\right)\right)$, the result follows.

\section{Proof of Proposition 11}

Noting that $\tilde{\lambda}=\frac{\lambda}{\mu}\left(1-e^{-\mu T}\right)<\lambda / \mu$ and using the result of Proposition 10 and the fact that $g(\tilde{\lambda})<1$, the store can be guaranteed to dominate whenever

$$
\frac{\lambda}{\mu}<\frac{\lambda}{\alpha+\lambda}-\frac{\alpha}{\alpha+\lambda} \frac{R}{\left(p^{*}-R\right)\left(1-F\left(p^{*}\right)\right)}
$$

or

$$
\mu>\frac{\lambda(\alpha+\lambda)}{\lambda-\frac{\alpha R}{\left(p^{*}-R\right)\left(1-F\left(p^{*}\right)\right)}}=\lambda \frac{\frac{\lambda}{\alpha}+1}{\frac{\lambda}{\alpha}-\frac{R}{\left(p^{*}-R\right)\left(1-F\left(p^{*}\right)\right)}}
$$

\section{Proof of Proposition 12}

Note that when $\alpha \rightarrow 0$, the payoff from the store is $\Pi_{S}=\pi_{1}$, whereas the payoff from the auction is $\pi(\tilde{\lambda}(T))=\sum_{N=0}^{\infty} e^{-\tilde{\lambda}(T)} \frac{\tilde{\lambda}(T)^{N}}{N !} \pi_{N}$. Also, since $\lambda \gg \mu, \tilde{\lambda}$ can be made sufficiently large so that $\pi(\tilde{\lambda})>\pi_{1}$ by setting a large auction closing time $T$. Therefore, using arguments similar to those in the proof of proposition 8 , one can establish that the auction dominates for low $\alpha$. 


\section{References}

ARNOLD, M.A. and LIPPMAN, S.A. (1995), "Optimal Selling Institutions: Auctions versus Sequential Search", Economic Inquiry, 33, 1-23.

BULOW, J. and ROBERTS, J. (1989), "The Simple Economics of Optimal Auctions", Journal of Political Economy, 97, 1060-1090.

BULOW, J. and KLEMPERER, P. (1996), "Auctions versus Negotiations", American Economic Review, 86, 180-194.

DE VANY, A. (1987), "Institutions for Stochastic Markets", Journal of Institutional and Theoretical Economics, 143, 91-103.

EHRMAN, C. and PETERS, M. (1993), "Sequential Selling Mechanisms", Economic Theory, 4, 237-253.

HARRIS, M. and RAVIV, A. (1981), "A Theory of Monopoly Pricing Schemes with Demand Uncertainty", American Economic Review, 71, 347-365.

LAZEAR, E.P. (1986), "Retail Pricing and Clearance Sales", American Economic Review, 76, 14-32.

LUCKING-REILEY, D. (2000), "Auctions on the Internet: What's Being Auctioned, and How?", Journal of Industrial Economics, 48, 227-252.

McAFEE, R.P. and McMILLAN, J. (1988), "Search Mechanisms", Journal of Economic Theory, 44, 99-123.

MILGROM, P.R. (1987), "Auction Theory", in: Bewley, T.F., Advances in Economic Theory, Cambridge University Press, New York, 1-32.

MILGROM, P.R. (1989), "Auctions and Bidding: A Primer", Journal of Economic Perspectives, 3, 3-22.

MYERSON, R. (1981), "Optimal Auction Design", Mathematics of Operations Research, 6, 58-73.

PINKER, E., SEIDMANN, A. and VAKRAT, Y. (2002), "The Design of Online Auctions: Business Issues and Current Research", Management Science, forthcoming.

RILEY, J. and SAMUELSON, W. (1981), "Optimal Auctions", American Economic Review, 71, 381-392.

RILEY, J. and ZECKHAUSER, R. (1983), "Optimal Selling Strategies: When to Haggle, When 
to Hold Firm", Quarterly Journal of Economics, 98, 267-289.

VICKREY, W. (1961), "Counterspeculation, Auctions, and Competitive Sealed Tenders", Journal of Finance, 16, 8-37.

WANG, R. (1993), "Auctions versus Posted-Price Selling", American Economic Review, 83, 838-851.

WANG, R. (1998), "Auctions versis Posted-Price Selling: The Case of Correlated Private Valuations", Canadian Journal of Economics, 31, 395-410. 